\title{
Parity Results for Certain Partition Functions and Identities Similar to Theta Function Identities
}

\author{
By Richard Blecksmith, John Brillhart, and Irving Gerst
}

Dedicated to Daniel Shanks on his seventieth birthday

Abstract. In this paper we give a collection of parity results for partition functions of the form

$$
\prod_{n \in S}\left(1-x^{n}\right)^{-1} \equiv \sum_{-\infty}^{\infty} x^{e(n)}(\bmod 2)
$$

and

$$
\prod_{n \in S}\left(1-x^{n}\right)^{-1} \equiv \sum_{-\infty}^{\infty}\left(x^{e(n)}+x^{f(n)}\right)(\bmod 2)
$$

for various sets of positive integers $S$, which are specified with respect to a modulus, and quadratic polynomials $e(n)$ and $f(n)$. Several identities similar to theta function identities, such as

$$
\prod_{\substack{n=1 \\ n \neq \pm(4,6,8,10)(\bmod 32)}}^{\infty}\left(1-x^{n}\right)=1+\sum_{n=1}^{\infty}(-1)^{n}\left(x^{n^{2}}+x^{2 n^{2}}\right),
$$

and its associated congruence

$$
\prod_{n \neq 1}^{\infty} \underset{n=1}{\infty}\left(1-x^{n}\right)^{-1} \equiv 1+\sum_{n=1}^{\infty}\left(x^{n^{2}}+x^{2 n^{2}}\right)(\bmod 2),
$$

are also proved.

1. Introduction. The question of determining the parity of the partition function $p(n)$ was raised by S. Ramanujan [7, pp. 1087, 1098], but has never been satisfactorily settled (see [6], [10]). Parkin and Shanks [8] have studied this question computationally and it is reasonable to conjecture on the basis of their statistical results that $p(n)$ is odd half the time.

Even though there is no simple formula known that gives the parity of $p(n)$, exact formulas do exist for other partition functions whose parts are the members of certain residue classes of a given modulus. In [1] two such results were established, namely:

$$
\prod_{n \in S}\left(1-x^{n}\right)^{-1} \equiv \sum_{-\infty}^{\infty} x^{e(n)}(\bmod 2)
$$

Received March 4, 1986; revised April 25, 1986 and June 25, 1986.

1980 Mathematics Subject Classification. Primary 10A45, 05A19.

Key words and phrases. Partition function parity, theta function analogues. 
for $S=\{n \in \mathbf{N}: n \equiv \pm(2,3,4,5,6,7)(\bmod 20)\}, e(n)=n(5 n+1) / 2$ and $S=$ $\{n \in \mathbf{N}: n \equiv \pm(1,2,5,6,8,9)(\bmod 20)\}, e(n)=n(5 n+3) / 2$ respectively. (See entries 4 and 5 in Table 1 below.)

In this paper we present a collection of parity results (obtained by using the methods discussed in [1]), which have been discovered over the last two years on an IBM Personal Computer and the Data General Eclipse S/230 at the Mathematics Department of the University of Arizona (see [2]).

2. Parity Results. In Table 1 we list results of the form (1). These can be proved using the Jacobi triple-product formula [5, p. 283]

$$
\prod_{\substack{n=1 \\ n=0, \pm(r-s)(\bmod 2 r)}}^{\infty}\left(1-x^{n}\right)=\sum_{-\infty}^{\infty}(-1)^{n} x^{r n^{2}+s n}
$$

by the same method used in proving Theorem 7.1 in [1]. (Entries 1 and 2 in Table 1 are well known and are given for completeness.)

TABLE 1

Parity Results of the First Kind

\begin{tabular}{cll}
\hline & \multicolumn{1}{c}{$S$} & \multicolumn{1}{c}{$e(n)$} \\
\hline 1. & $n \equiv 1(\bmod 2)$ & $n(3 n+1) / 2$ \\
2. & $n \neq \equiv 0(\bmod 4)$ & $n(2 n+1)$ \\
3. & $n \neq \equiv 0, \pm 3(\bmod 12)$ & $n(3 n+2)$ \\
4. & $n \neq \equiv 0, \pm 1, \pm 8, \pm 9,10(\bmod 20)$ & $n(5 n+1) / 2$ \\
5. & $n \neq \equiv 0, \pm 3, \pm 4, \pm 7,10(\bmod 20)$ & $n(5 n+3) / 2$ \\
\hline
\end{tabular}

Table 2 contains a collection of results of the form

$$
\prod_{n \in S}\left(1-x^{n}\right)^{-1} \equiv \sum_{-\infty}^{\infty}\left(x^{e(n)}+x^{f(n)}\right)(\bmod 2) \text {. }
$$

TABLE 2

Parity Results of the Second Kind

\begin{tabular}{llll}
\hline & \multicolumn{1}{c}{$S$} & \multicolumn{1}{c}{$e(n)$} & \multicolumn{1}{c}{$f(n)$} \\
\hline 1. & $n \neq \equiv 0, \pm 1(\bmod 8)$ & $n(12 n+5)$ & $(3 n+1)(4 n+3)$ \\
2. & $n \neq \equiv 0, \pm 3(\bmod 8)$ & $n(12 n+1)$ & $(3 n+1)(4 n+1)$ \\
3. & $n \not \equiv 0, \pm 2(\bmod 10)$ & $n(15 n+7) / 2$ & $(3 n+2)(5 n+1) / 2$ \\
4. & $n \not \equiv 0, \pm 4(\bmod 10)$ & $n(15 n+1) / 2$ & $(3 n+1)(5 n+2) / 2$ \\
5. & $n \neq \equiv 0, \pm 3, \pm 4(\bmod 12)$ & $9 n^{2}$ & $(3 n+1)^{2}$ \\
6. & $n \neq \equiv 0, \pm 1, \pm 12, \pm 13,14(\bmod 28)$ & $n(21 n+5) / 2$ & $(3 n+1)(7 n+3) / 2$ \\
7. & $n \neq \equiv 0, \pm 3, \pm 8, \pm 11,14(\bmod 28)$ & $n(21 n+1) / 2$ & $(3 n+1)(7 n+2) / 2$ \\
8. & $n \neq \equiv 0, \pm 4, \pm 5, \pm 9,14(\bmod 28)$ & $n(21 n+11) / 2$ & $(3 n+2)(7 n+1) / 2$
\end{tabular}

Observe that 5 in Table 2 can be simplified to give the right-hand side

$$
1+x+\sum_{n=1}^{\infty}\left[x^{(3 n-1)^{2}}+x^{(3 n+1)^{2}}\right](\bmod 2) \text {. }
$$


In our search for new $(\bmod 2)$ congruences, all possible residue classes were tried for moduli up to 16 as well as symmetrically placed classes for moduli up to 30 . We should observe that many of the results of this paper can be expressed alternatively in the language of partitions in an obvious way, but we have not done this in this paper.

The method of proving the results in Table 2 will be illustrated by proving identity 3.

Proof. From [5, p. 284], we have the congruence

$$
\prod_{n=1}^{\infty}\left(1-x^{2 n-1}\right)^{2}\left(1-x^{2 n}\right)=1+2 \sum_{n=1}^{\infty}(-1)^{n} x^{n^{2}} \equiv 1(\bmod 2),
$$

which can sometimes be used to invert a product $(\bmod 2)$, since, in the product on the left, each odd exponent occurs twice and each even exponent once. In the present case we have the following $(\bmod 2)$ identity (in which the reciprocal product on the left generates partitions whose parts are the integers in the specified residue classes and the product on the right generates the excess of the partitions with an even number of distinct parts over the partitions with an odd number of distinct parts [5, p. 287]):

$$
\prod_{\substack{n=1 \\ n \equiv \pm 1, \pm 3, \pm 4,5(\bmod 10)}}^{\infty}\left(1-x^{n}\right)^{-1} \equiv \prod_{\substack{n=1 \\ n \equiv 0, \pm 1, \pm 2, \pm 3,5(\bmod 10)}}^{\infty}\left(1-x^{n}\right)(\bmod 2) .
$$

(Here the modulus is even, so in the cross multiplication the odd exponents $\pm 1, \pm 3$, and $5(\bmod 10)$ occur twice and the even exponents $0, \pm 2$, and \pm 4 occur once, as they should.)

To expand the product on the right we use the following result [4, p. 287] (see [3] for the history of the general product from which (5) was derived):

$$
\prod_{n \in S_{1}}\left(1-x^{n}\right)=\sum_{-\infty}^{\infty} x^{\left(3 n^{2}+n\right) M / 2}\left(x^{-3 k n}-x^{3 k n+k}\right),
$$

where $M$ and $k$ are integers such that $0<2 k<M$ and

$$
S_{1}=\{n \in \mathbf{N}: n \equiv 0, \pm k, \pm(M-2 k), \pm(M-k), M(\bmod 2 M)\} .
$$

The (mod 2) result follows from (4) and (5) with $M=5$ and $k=2$.

Note that the $(\bmod 2)$ congruence in $(3)$ can also be obtained immediately from the formula of Euler [5, p. 277, (19.4.7)]

$$
\prod_{n=1}^{\infty}\left(1+x^{n}\right)\left(1-x^{2 n-1}\right)=1
$$

3. New Identities and Associated Parity Results. The remainder of this paper will be devoted to proving some parity results of a particularly simple form, which are derived from identities similar to theta function identities.

THEOREM 1.

$$
\begin{aligned}
\prod_{\substack{n=1 \\
n \neq \pm(4,6,8,10)(\bmod 32)}}^{\infty}\left(1-x^{n}\right) & =1+\sum_{n=1}^{\infty}(-1)^{n}\left(x^{n^{2}}+x^{2 n^{2}}\right) . \\
\prod_{\substack{n=1 \\
n \neq \pm(2,8,12,14)(\bmod 32)}}^{\infty}\left(1-x^{n}\right) & =\sum_{n=1}^{\infty}(-1)^{n}\left(x^{2 n^{2}-1}-x^{n^{2}-1}\right) .
\end{aligned}
$$


Proof. (a) Rewriting (3), we obtain

$$
\prod_{n=1}^{\infty}\left(1-x^{n}\right)\left(1-x^{2 n-1}\right)=1+2 \sum_{n=1}^{\infty}(-1)^{n} x^{n^{2}}
$$

Also, recall the familiar identity [5, p. 284]

$$
\prod_{n=1}^{\infty}\left(1-x^{n}\right)=\sum_{-\infty}^{\infty}(-1)^{n} x^{\left(3 n^{2}+n\right) / 2}
$$

If we replace $x$ by $x^{2}$ in (6) and add the resulting identity to (6), we obtain

$$
\begin{aligned}
1+ & \sum_{n=1}^{\infty}(-1)^{n}\left(x^{n^{2}}+x^{2 n^{2}}\right) \\
& =\frac{1}{2}\left[\prod_{n=1}^{\infty}\left(1-x^{n}\right)\left(1-x^{2 n-1}\right)+\prod_{n=1}^{\infty}\left(1-x^{2 n}\right)\left(1-x^{2 n-1}\right)\left(1+x^{2 n-1}\right)\right] \\
& =\frac{1}{2} \prod_{n=1}^{\infty}\left(1-x^{2 n-1}\right)\left\{\prod_{n=1}^{\infty}\left(1-x^{n}\right)+\prod_{n=1}^{\infty}\left[1-(-x)^{n}\right]\right\},
\end{aligned}
$$

which by (7) equals

$$
\frac{1}{2} \prod_{n=1}^{\infty}\left(1-x^{2 n-1}\right)\left[\sum_{-\infty}^{\infty}(-1)^{n} x^{\left(3 n^{2}+n\right) / 2}+\sum_{-\infty}^{\infty}(-1)^{n}(-1)^{\left(3 n^{2}+n\right) / 2} x^{\left(3 n^{2}+n\right) / 2}\right] .
$$

Thus, we have

$$
\begin{aligned}
& 1+\sum_{n=1}^{\infty}(-1)^{n}\left(x^{n^{2}}+x^{2 n^{2}}\right) \\
& \quad=\frac{1}{2} \prod_{n=1}^{\infty}\left(1-x^{2 n-1}\right) \sum_{-\infty}^{\infty}(-1)^{n}\left[1+(-1)^{\left(3 n^{2}+n\right) / 2}\right] x^{\left(3 n^{2}+n\right) / 2} .
\end{aligned}
$$

Now $\left(3 n^{2}+n\right) / 2$ is even when $n \equiv 0,1(\bmod 4)$ and odd when $n \equiv 2,3(\bmod 4)$.

Thus, the right-hand side becomes

$$
\prod_{n=1}^{\infty}\left(1-x^{2 n-1}\right) \sum_{\substack{-\infty \\ n \equiv 0,1(\bmod 4)}}^{\infty}(-1)^{n} x^{\left(3 n^{2}+n\right) / 2} .
$$

Replacing $n$ by $4 n$ and $4 n+1$ in the summation, we obtain

$$
\prod_{n=1}^{\infty}\left(1-x^{2 n-1}\right) \sum_{-\infty}^{\infty}\left(x^{24 n^{2}+2 n}-x^{24 n^{2}+14 n+2}\right),
$$

which, by (5) with $M=16, k=2$, gives

$$
\prod_{n=1}^{\infty}\left(1-x^{2 n-1}\right) \prod_{n \equiv 0, \pm 2, \pm 12, \pm 14,16(\bmod 32)}^{\infty}\left(1-x^{n}\right) .
$$

(b) If we replace $x$ by $x^{2}$ in (6) and subtract (6) from the resulting identity, we obtain

$$
\begin{aligned}
& \prod_{n=1}^{\infty}(-1)^{n}\left(x^{2 n^{2}-1}-x^{n^{2}-1}\right) \\
& \quad=\frac{1}{2 x} \prod_{n=1}^{\infty}\left(1-x^{2 n-1}\right)\left[\prod_{n=1}^{\infty}\left(1-x^{2 n}\right)\left(1+x^{2 n-1}\right)-\prod_{n=1}^{\infty}\left(1-x^{n}\right)\right] \\
& \quad=\frac{1}{2 x} \prod_{n=1}^{\infty}\left(1-x^{2 n-1}\right)\left[\prod_{n=1}^{\infty}\left[1-(-x)^{n}\right]-\prod_{n=1}^{\infty}\left(1-x^{n}\right)\right] .
\end{aligned}
$$


As in (8) we have

$$
\begin{gathered}
\frac{1}{2 x} \prod_{n=1}^{\infty}\left(1-x^{2 n-1}\right) \sum_{-\infty}^{\infty}(-1)^{n}\left[(-1)^{\left(3 n^{2}+n\right) / 2}-1\right] x^{\left(3 n^{2}+n\right) / 2} \\
=\frac{1}{x} \prod_{n=1}^{\infty}\left(1-x^{2 n-1}\right) \sum_{\substack{-\infty \\
n}}^{\infty}(-1)^{n+1} x^{\left(3 n^{2}+n\right) / 2} .
\end{gathered}
$$

Replacing $n$ by $4 n+2$ and $4 n+3$ in the summation, we obtain

$$
\prod_{n=1}^{\infty}\left(1-x^{2 n-1}\right) \sum_{-\infty}^{\infty}\left(x^{24 n^{2}+38 n+14}-x^{24 n^{2}+26 n+6}\right),
$$

which, using (5) with $M=16$ and $k=6$, equals

$$
\prod_{n=1}^{\infty}\left(1-x^{2 n-1}\right) \prod_{\substack{n=1 \\ n \equiv 0, \pm 4, \pm 6, \pm 10,16(\bmod 32)}}^{\infty}\left(1-x^{n}\right),
$$

where $n$ was replaced by $n-1$ in the first sum.

Corollary 1.

(a)

$$
\begin{array}{r}
\prod_{\substack{n=1 \\
n \neq \pm(4,6,8,10)(\bmod 32)}}^{\infty}\left(1-x^{n}\right)+x \prod_{n \neq \pm(2,8,12,14)(\bmod 32)}^{\infty}\left(1-x^{n}\right) \\
=1+2 \sum_{n=1}^{\infty}(-1)^{n} x^{2 n^{2}}=\prod_{n=1}^{\infty}\left(1-x^{2 n}\right)\left(1-x^{4 n-2}\right) .
\end{array}
$$

(b)

$$
\begin{aligned}
\prod_{\substack{n=1 \\
n \neq \pm(4,6,8,10)(\bmod 32)}}^{\infty}\left(1-x^{n}\right)-x & \prod_{\substack{n=1 \\
n \neq \pm(2,8,12,14)(\bmod 32)}}^{\infty}\left(1-x^{n}\right) \\
=1+2 \sum_{n=1}^{\infty}(-1)^{n} x^{n^{2}} & =\prod_{n=1}^{\infty}\left(1-x^{n}\right)\left(1-x^{2 n-1}\right) .
\end{aligned}
$$

(c)

$$
\begin{gathered}
\prod_{\substack{n=1 \\
n \neq 0, \pm 2, \pm 12, \pm 14,16(\bmod 32)}}^{\infty}\left(1-x^{n}\right)^{-1} \\
-x \prod_{\substack{n=1 \\
n \neq 0, \pm 4, \pm 6, \pm 10,16(\bmod 32)}}^{\infty}\left(1-x^{n}\right)^{-1}=1 .
\end{gathered}
$$

Proof. (a) The first equality follows from Theorems 1(a) and 1(b). The second equality is (6) with $x$ replaced by $x^{2}$.

(b) The first equality follows from Theorems 1 (a) and 1(b). The second is (6).

(c) This equation follows from Corollary 1(b).

\section{THEOREM 2.}

(a) $\prod_{\substack{n=1 \\ n \neq 0, \pm 2, \pm 12, \pm 14,16(\bmod 32)}}^{\infty}\left(1-x^{n}\right)^{-1} \equiv 1+\sum_{n=1}^{\infty}\left(x^{n^{2}}+x^{2 n^{2}}\right)(\bmod 2)$.

(b) $\prod_{\substack{n=1 \\ n \neq 0, \pm 4, \pm 6, \pm 10,16(\bmod 32)}}^{\infty}\left(1-x^{n}\right)^{-1} \equiv \sum_{n=1}^{\infty}\left(x^{n^{2}-1}+x^{2 n^{2}-1}\right)(\bmod 2)$. 
(c)

$$
\begin{gathered}
\prod_{\substack{n=1 \\
n \neq 0, \pm 2, \pm 12, \pm 14,16(\bmod 32)}}^{\infty}\left(1-x^{n}\right)^{-1} \\
+x \prod_{\substack{n=1 \\
n \neq 0, \pm 4, \pm 6, \pm 10,16(\bmod 32)}}^{\infty}\left(1-x^{n}\right)^{-1} \equiv 1(\bmod 2) .
\end{gathered}
$$

Proof. (a) From Theorem 1(a) we have

$$
\begin{aligned}
1+ & \sum_{n=1}^{\infty}\left(x^{n^{2}}+x^{2 n^{2}}\right) \equiv \prod_{\substack{n=1 \\
n \neq \pm(4,6,8,10)(\bmod 32)}}^{\infty}\left(1-x^{n}\right) \\
& =\prod_{n=1}^{\infty}\left(1-x^{2 n-1}\right) \prod_{\substack{n=0, \pm 1, \pm 6, \pm 7,8(\bmod 16) \\
n=1}}^{\infty}\left(1-x^{2 n}\right) \\
& \equiv \prod_{n=1}^{\infty}\left(1-x^{2 n-1}\right)^{-1} \prod_{\substack{n=1 \\
n \neq 0, \pm 1, \pm 6, \pm 7,8(\bmod 16)}}^{\infty}\left(1-x^{2 n}\right)^{-1} \\
= & \prod_{\substack{n=1 \\
n \neq 0, \pm 2, \pm 12, \pm 14,16(\bmod 32)}}^{\infty}\left(1-x^{n}\right)^{-1}(\bmod 2),
\end{aligned}
$$

using the $(\bmod 2)$ reciprocation method derived from $(3)$.

(b) From Theorem 1(b) we have

$$
\begin{gathered}
\sum_{n=1}^{\infty}\left(x^{2 n^{2}-1}+x^{n^{2}-1}\right) \equiv \prod_{\substack{n=1 \\
n \neq \pm(2,8,12,14)(\bmod 32)}}^{\infty}\left(1-x^{n}\right) \\
=\prod_{n=1}^{\infty}\left(1-x^{2 n-1}\right) \prod_{\substack{n \equiv 0, \pm 2, \pm 3, \pm 5,8(\bmod 16) \\
n}}^{\infty}\left(1-x^{2 n}\right) \\
\equiv \prod_{n=1}^{\infty}\left(1-x^{2 n-1}\right)^{-1} \prod_{\substack{n=1 \\
n \neq 0, \pm 2, \pm 3, \pm 5,8(\bmod 16)}}^{\infty}\left(1-x^{2 n}\right)^{-1} \\
=\prod_{n \neq 0, \pm 4, \pm 6, \pm 10,16(\bmod 32)}^{\infty}\left(1-x^{n}\right)^{-1} .
\end{gathered}
$$

(c) This result follows from the second equality of Corollary 1(c), dividing first by the product $\prod_{n=1}^{\infty}\left(1-x^{2 n-1}\right)$.

THEOREM 3.
(a)
$\prod_{\substack{n=1 \\ n \neq \pm(2,4,5,6,7,8)(\bmod 24)}}^{\infty}\left(1-x^{n}\right)=1+\sum_{n=1}^{\infty}(-1)^{n}\left(x^{n^{2}}+x^{3 n^{2}}\right)$.
(b)

$$
\prod_{n= \pm(1,4,6,8,10,11)(\bmod 24)}^{\infty}
$$$$
\left(1-x^{n}\right)=\sum_{n=1}^{\infty}(-1)^{n}\left(x^{3 n^{2}-1}-x^{n^{2}-1}\right) .
$$ 
Proof. (a) If in (3) we replace $x$ by $x^{3}$ and add the resulting identity to (3), we obtain

$$
\begin{aligned}
1+\sum_{n=1}^{\infty}(-1)^{n}\left(x^{n^{2}}+x^{3 n^{2}}\right) & \\
= & \frac{1}{2}\left[\prod_{n=1}^{\infty}\left(1-x^{2 n-1}\right)^{2}\left(1-x^{2 n}\right)+\prod_{n=1}^{\infty}\left(1-x^{6 n-3}\right)^{2}\left(1-x^{6 n}\right)\right] \\
= & \frac{1}{2} \prod_{n=1}^{\infty}\left(1-x^{6 n^{-3}}\right)\left[\prod_{n=1}^{\infty}\left(1-x^{6 n-5}\right)^{2}\left(1-x^{6 n-3}\right)\left(1-x^{6 n-1}\right)^{2}\left(1-x^{6 n-4}\right)\right. \\
& \left.\quad \times\left(1-x^{6 n-2}\right)\left(1-x^{6 n}\right)+\prod_{n=1}^{\infty}\left(1-x^{6 n-3}\right)\left(1-x^{6 n}\right)\right] .
\end{aligned}
$$

Using (5) with $M=3, k=1$ on the first product inside the bracket, we get

$$
\frac{1}{2} \prod_{n=1}^{\infty}\left(1-x^{6 n-3}\right)\left\{\sum_{-\infty}^{\infty}\left(x^{\left(9 n^{2}-3 n\right) / 2}-x^{\left(9 n^{2}+9 n+2\right) / 2}\right)+\prod_{n=1}^{\infty}\left[1-\left(x^{3}\right)^{n}\right]\right\} \text {. }
$$

Now, replacing $n$ by $-n$ in the first sum and using (7) gives

$$
\begin{gathered}
\frac{1}{2} \prod_{n=1}^{\infty}\left(1-x^{6 n-3}\right)\left[\sum_{-\infty}^{\infty}\left(x^{\left(9 n^{2}+3 n\right) / 2}-x^{\left(9 n^{2}+9 n+2\right) / 2}\right)+\sum_{-\infty}^{\infty}(-1)^{n} x^{\left(9 n^{2}+3 n\right) / 2}\right] \\
=\frac{1}{2} \prod_{n=1}^{\infty}\left(1-x^{6 n-3}\right)\left\{\sum_{-\infty}^{\infty}\left[1+(-1)^{n}\right] x^{\left(9 n^{2}+3 n\right) / 2}-\sum_{-\infty}^{\infty} x^{\left(9 n^{2}+9 n+2\right) / 2}\right\} .
\end{gathered}
$$

If we separate the indices of the two sums into even and odd values, the first sum is 0 when $n$ is odd, and in the second sum, if we write

(9) $f(n)=\left(9 n^{2}+9 n+2\right) / 2$, then $f(2 n)=f(-2 n-1)=18 n^{2}+9 n+1$, we obtain

$$
\prod_{n=1}^{\infty}\left(1-x^{6 n-3}\right) \sum_{-\infty}^{\infty}\left(x^{18 n^{2}+3 n}-x^{18 n^{2}+9 n+1}\right) .
$$

Thus, using (5) with $M=12, k=1$ we have

$$
\prod_{n=1}^{\infty}\left(1-x^{6 n-3}\right) \prod_{\substack{n=1 \\ n \equiv 0, \pm 1, \pm 10, \pm 11,12(\bmod 24)}}^{\infty}\left(1-x^{n}\right) .
$$

(b) If we replace $x$ by $x^{3}$ in (3) and subtract (3) from the resulting identity, we obtain

$$
\begin{aligned}
& \sum_{n=1}^{\infty}(-1)^{n}\left(x^{3 n^{2}-1}-x^{n^{2}-1}\right) \\
& =\frac{1}{2 x}\left[\prod_{n=1}^{\infty}\left(1-x^{6 n-3}\right)^{2}\left(1-x^{6 n}\right)-\prod_{n=1}^{\infty}\left(1-x^{2 n-1}\right)^{2}\left(1-x^{2 n}\right)\right] \\
& =\frac{1}{2 x} \prod_{n=1}^{\infty}\left(1-x^{6 n-3}\right)\left[\prod_{n=1}^{\infty}\left(1-x^{6 n-3}\right)\left(1-x^{6 n}\right)\right. \\
& -\prod_{n=1}^{\infty}\left(1-x^{6 n-5}\right)^{2}\left(1-x^{6 n-3}\right)\left(1-x^{6 n-1}\right)^{2} \\
& \left.\quad \times\left(1-x^{6 n-4}\right)\left(1-x^{6 n-2}\right)\left(1-x^{6 n}\right)\right]
\end{aligned}
$$


As in part (a), we can write this expression as

$$
\begin{aligned}
& =\frac{1}{2 x} \prod_{n=1}^{\infty}\left(1-x^{6 n-3}\right)\left[\sum_{-\infty}^{\infty}(-1)^{n} x^{\left(9 n^{2}+3 n\right) / 2}-\sum_{-\infty}^{\infty}\left(x^{\left(9 n^{2}+3 n\right) / 2}-x^{\left(9 n^{2}+9 n+2\right) / 2}\right)\right] \\
& =\frac{1}{2 x} \prod_{n=1}^{\infty}\left(1-x^{6 n-3}\right)\left\{\sum_{-\infty}^{\infty}\left[(-1)^{n}-1\right] x^{\left(9 n^{2}+3 n\right) / 2}+\sum_{-\infty}^{\infty} x^{\left(9 n^{2}+9 n+2\right) / 2}\right\} .
\end{aligned}
$$

Separating the indices of the two sums into even and odd values, we find the first sum is 0 when $n$ is even, and in the second sum, using (9), we obtain

$$
\prod_{n=1}^{\infty}\left(1-x^{6 n-3}\right) \sum_{-\infty}^{\infty}\left(x^{18 n^{2}+9 n}-x^{18 n^{2}+21 n+5}\right) .
$$

Replacing $n$ by $-n$ in the first sum and using (5) with $M=12, k=5$, we have

$$
\prod_{n=1}^{\infty}\left(1-x^{6 n-3}\right) \prod_{\substack{n=1 \\ n \equiv 0, \pm 2, \pm 5, \pm 7,12(\bmod 24)}}^{\infty}\left(1-x^{n}\right) .
$$

Corollary 2.

(a)

$$
\begin{gathered}
\prod_{\substack{n=1 \\
n \neq \pm(2,4,5,6,7,8)(\bmod 24)}}^{\infty}\left(1-x^{n}\right)+x \quad \prod_{\substack{n=1 \\
n \neq \pm(1,4,6,8,10,11)(\bmod 24)}}^{\infty}\left(1-x^{n}\right) \\
=1+2 \sum_{n=1}^{\infty}(-1)^{n} x^{3 n^{2}}=\prod_{n=1}^{\infty}\left(1-x^{3 n}\right)\left(1-x^{6 n-3}\right) .
\end{gathered}
$$

(b)

$$
\begin{aligned}
\prod_{n=1}^{\infty}\left(1-x^{n}\right)-x & \prod_{n=1}^{\infty}\left(1-x^{n}\right) \\
=1+2 \sum_{n=1}^{\infty}(-1)^{n} x^{n^{2}}=\prod_{n=1}^{\infty}\left(1-x^{n}\right)\left(1-x^{2 n-1}\right) . &
\end{aligned}
$$

(c) $\prod_{n \in S_{1}}\left(1-x^{n}\right)^{-1}-x \prod_{n \in S_{2}}\left(1-x^{n}\right)^{-1}=1$,

where

$$
S_{1}=\left\{n \in \mathbf{N}: n \equiv \pm\left(1,2,3,4,5^{2}, 6,7^{2}, 8,9,11\right)(\bmod 24)\right\}
$$

and

$$
S_{2}=\left\{n \in \mathbf{N}: n \equiv \pm\left(1^{2}, 3,4,5,6,7,8,9,10,11^{2}\right)(\bmod 24)\right\} .
$$

Proof. (a), (b) These identities follow from (6) and Theorems 3(a) and 3(b).

(c) This follows from Corollary 2(b) by dividing through first by $\prod_{n=1}^{\infty}\left(1-x^{n}\right)$ and then by $\prod_{n=1}^{\infty}\left(1-x^{2 n-1}\right)$.

Remark. Corollary 2(c) can also be proved by separating the index values in the sum on the right side of (7) into the even and odd forms $2 n$ and $-2 n-1$. Thus,

$$
\begin{aligned}
\prod_{n=1}^{\infty}\left(1-x^{n}\right)= & \sum_{-\infty}^{\infty} x^{6 n^{2}+n}-x \sum_{-\infty}^{\infty} x^{6 n^{2}+5 n} \\
= & \sum_{\substack{n=1 \\
n \equiv 0(\bmod 12)}}^{\infty}\left(1-x^{n}\right) \prod_{\substack{n=1 \\
n \equiv \pm(\bmod 12)}}^{\infty}\left(1+x^{n}\right) \\
& -x \prod_{n=0(\bmod 12)}^{\infty}\left(1-x^{n}\right) \prod_{\substack{n=1 \\
n \equiv \pm 1(\bmod 12)}}^{\infty}\left(1+x^{n}\right),
\end{aligned}
$$


using the formula $[5$, p. 283]

$$
\sum_{-\infty}^{\infty} x^{r n^{2}+s n}=\prod_{n=1}^{\infty}\left(1-x^{2 r n}\right)\left(1+x^{2 r n-r+s}\right)\left(1+x^{2 r n-r-s}\right) .
$$

Dividing through by $\prod_{n=1}^{\infty}\left(1-x^{n}\right)$ gives

$$
\begin{aligned}
& {\left[\prod_{\substack{n=1 \\
n \equiv \pm 5(\bmod 12)}}^{\infty}\left(1+x^{n}\right) / \prod_{\substack{n=1 \\
n \neq 0(\bmod 12)}}^{\infty}\left(1-x^{n}\right)\right]} \\
& -x\left[\prod_{\substack{n=1 \\
n \equiv \pm 1(\bmod 12)}}^{\infty}\left(1+x^{n}\right) / \prod_{\substack{n=1 \\
n \neq 0(\bmod 12)}}^{\infty}\left(1-x^{n}\right)\right]=1 .
\end{aligned}
$$

The proof is completed by canceling the numerators of the two fractions into the denominator factor

$$
\begin{aligned}
& \prod_{\substack{n=1 \\
n \equiv \pm 2(\bmod 12)}}^{\infty}\left(1-x^{n}\right) \\
= & \prod_{\substack{n=1 \\
n \equiv \pm 2(\bmod 24)}}^{\infty}\left(1-x^{n}\right) \prod_{\substack{n=1 \\
n \equiv \pm 5(\bmod 12)}}^{\infty}\left(1-x^{n}\right) \prod_{\substack{n=1 \\
n \equiv \pm 5(\bmod 12)}}^{\infty}\left(1+x^{n}\right) \\
= & \prod_{\substack{n=1 \\
n \equiv \pm 1(\bmod 12)}}^{\infty}\left(1+x^{n}\right) \prod_{\substack{n=1 \\
n \equiv \pm 1(\bmod 12)}}^{\infty}\left(1-x^{n}\right) \prod_{\substack{n=1 \\
n \equiv \pm 10(\bmod 24)}}^{\infty}\left(1-x^{n}\right) .
\end{aligned}
$$

Other more complicated identities of this kind can be derived from (7) by separating the indices on the right side into more than two classes.

\section{TheOREM 4.}
(a)

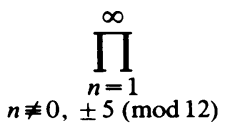$$
\left(1-x^{n}\right)^{-1} \equiv 1+\sum_{n=1}^{\infty}\left(x^{n^{2}}+x^{3 n^{2}}\right)(\bmod 2)
$$
(b)

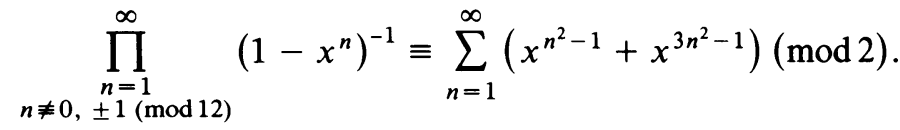
(c)

$$
\prod_{\substack{n=1 \\ n \neq 0, \pm 5(\bmod 12)}}^{\infty}\left(1-x^{n}\right)^{-1}+x \prod_{\substack{n=1 \\ n \neq 0, \pm 1(\bmod 12)}}^{\infty}\left(1-x^{n}\right)^{-1} \equiv 1(\bmod 2) .
$$

Proof. (a) From Theorem 3(a) and (3) we have

$$
\begin{gathered}
1+\sum_{n=1}^{\infty}\left(x^{n^{2}}+x^{3 n^{2}}\right) \equiv \prod_{\substack{n=1 \\
n \equiv 0, \pm 1, \pm 3, \pm 9, \pm 10, \pm 11,12(\bmod 24)}}^{\infty}\left(1-x^{n}\right) \\
\equiv \prod_{\substack{n=1 \\
n \equiv \pm(1,2,3,4,6,8,9,10,11)(\bmod 24)}}^{\infty}\left(1-x^{n}\right)^{-1}=\prod_{\substack{n=1 \\
n \neq 0, \pm 5(\bmod 12)}}^{\infty}\left(1-x^{n}\right)^{-1},
\end{gathered}
$$

where we have used in the reciprocation of the infinite product the fact that

$$
1-x^{24 n \pm 10} \equiv\left(1-x^{12 n \pm 5}\right)^{2}=\left(1-x^{24 n \pm 5}\right)^{2}\left(1-x^{24 n \pm 7}\right)^{2}(\bmod 2) .
$$


(b) This follows from Theorems 4(a) and 3(c).

(c) This follows from Theorems 4(a) and (b) or Corollary 2(c), using (mod 2) calculations like those in the proof of Theorem 4(a).

In conclusion, we would like to thank Michael Filaseta for his comments about congruence (3). Also we would like to express our appreciation to George Andrews for sending us proofs of Theorems 2 and 4, and Michael Hirschhorn for sending us proofs of the same theorems and several other $(\bmod 2)$ results. The methods used in these proofs were different from our own.

Department of Mathematics

Northern Illinois University

DeKalb, Illinois 60115

Department of Mathematics

University of Arizona

Tucson, Arizona 85721

Department of Applied Mathematics and Statistics

SUNY at Stony Brook

Stony Brook, New York 11794

1. R. Blecksmith, John Brillhart \& Irving Gerst, "A computer-assisted investigation of Ramanujan pairs," Math. Comp., v. 46, 1986, pp. 731-749.

2. R. BLECKSMITH, John BRILlhart \& IRVING Gerst, "Partition function parity theorems and theta function identity analogues," Abstracts Amer. Math. Soc., v. 7, 1986, p. 225.

3. L. Carlitz \& M. V. Subbarao, "A simple proof of the quintuple product identity," Proc. Amer. Math. Soc., v. 32, 1972, pp. 42-44.

4. B. GoRDON, "Some identities in combinatorial analysis," Quart. J. Math. Oxford Ser. (2), v. 12, 1961, pp. 285-290, MR 25 \#2340.

5. G. H. Hardy \& E. M. WRIGHT, An Introduction to the Theory of Numbers, 4th ed., Oxford Univ. Press, Oxford, 1965.

6. M. D. Hirschrorn, "On the residue $\bmod 2$ and $\bmod 4$ of $p(n)$," Acta Arith., v. 38, 1980, pp. 105-109.

7. P. A. MacMahon, Collected Papers, vol. 1, MIT Press, Cambridge, Mass., 1978.

8. T. R. Parkin \& D. Shanks, "On the distribution of parity in the partition function," Math. Comp., v. 21,1967 , pp. $466-480$.

9. L. J. SLATER, "Further identities of the Rogers-Ramanujan type," Proc. London Math. Soc. (2), v. 54, 1952, pp. 147-167. MR 14, 138.

10. M. V. SubbaraO, "Some remarks on the partition function," Amer. Math. Monthly, v. 73, 1966, pp. 851-854. 